\title{
ORIGINAL ARTICLE \\ Bedside prediction of the progress of pressure ulcer healing in patients with spinal cord injury using the 'Decu-stick'
}

\author{
FWA Van Asbeck ${ }^{1,2}$ and MWM Post ${ }^{2,3}$
}

Study design: This is a prospective cohort study.

Objectives: The objective of this study was to predict the progress of healing of pressure ulcers (PUs) in spinal cord injury (SCl) patients after the first 4 weeks.

Setting: The study was conducted in a specialized SCl rehabilitation unit in The Netherlands.

Methods: Weekly measurements of length, width and depth/undermining of grades II-IV PUs under sacrum or ischial tuberosity in SCI patients with the 'Decu-stick' were taken. The speed of reduction of the greatest dimension in the first 4 weeks of the granulationepithelization (G-E) phase was compared with the speed of reduction of this dimension after week 4 until the end of observation.

Results: Fifty-one PUs in 45 patients were measured. During the first 4 weeks of the G-E phase, the greatest dimension of 23/51 PUs reduced with a speed of $\geqslant 0.5 \mathrm{~cm}$ per week. In 22 of these $23 \mathrm{PUs}$, this speed remained $\geqslant 0.5$ from week 4 until the end of observation (weeks 5-22). Closure: 21 patients (pts); operation: 2 pts. Of $28 / 51 \mathrm{PUs}$, this dimension reduced with $<0.5 \mathrm{~cm}$ per week. In 27/28 PUs, this speed remained <0.5 from week 4 until the end of observation (weeks 9-37). Closure: 6 pts; operation: 16 pts; discharge with open ulcer: 6 pts.

Conclusion: Measurement of PUs in SCl patients with the 'Decu-stick' provides a reliable, quick, cheap and easy-to-learn bedside method to predict the progress of healing in $\mathrm{PUs}$ in $\mathrm{SCl}$ patients after 4 weeks of conservative treatment with a positive predictive value of 0.95 and an negative predictive value of 0.96 . This provides a scientific basis for the decision on operative or alternative conservative treatment.

Spinal Cord (2015) 53, 539-543; doi:10.1038/sc.2015.40; published online 10 March 2015

\section{INTRODUCTION}

Pressure ulcers (PUs) are a common secondary health condition in spinal cord injury (SCI) with a prevalence ranging from 15.2 to $42.5 \%{ }^{1-4}$ Treatment can be conservative or surgical. Operative closure is usually performed by covering the ulcer using a rotation flap. As the number of possible rotation flaps in one patient is limited and the life expectancy of SCI patients is long, conservative treatment is preferred. However, this can take a long time, it is a burden to patients and it is not always successful. To timely change from conservative treatment to operative treatment, prediction of the progress of healing of a PU under conservative treatment is required.

However, we found only one study on the prediction of healing of PUs in SCI. ${ }^{5}$ This study used stereophotogrammetric digital imaging, which is unable to assess undermining. A study on wound measurement not limited to PUs reported that a $20-40 \%$ reduction of wound area in 2 and 4 weeks, respectively, is likely to be a reliable predictive indicator of healing but specifically for venous legs ulcers. ${ }^{6}$ A review on mathematical formulas used to assess the rate of wound healing in chronic venous, neuropathic, pressure or ischemic wounds stated that 'linear advancement of wound margins toward the center of the wound [..] after 4 weeks of healing may prove to be of value in predicting time to complete healing.' ${ }^{7}$
Prediction of the progress of healing is based on wound measurement. For this measurement, guidelines recommend to regularly measure length, width and depth of the wound to evaluate the response to treatment. ${ }^{1,8}$ Length measurement should be along the longest dimension of the wound and width measurement should be along the maximum dimension perpendicular to the length axis. The depth of the wound should be measured from the deepest point to the imaginary surface in continuity with the edges of the wound. ${ }^{1}$ PUs are typically surface, crater or undermining wounds with sometimes tunneling and sinus tract formation. Thus, assessment of wound depth should also include evaluation of sinus tracts, which may communicate with deeper structures. ${ }^{1}$

In a review in 2010 of instruments to measure the healing of PUs, only 11 methods were found that have been evaluated for their clinimetric properties and described in more than one article. ${ }^{9}$ Since then, only one additional instrument has been published. ${ }^{10}$ Of these 12 instruments, 5 measured the surface and 3 measured the volume of PUs. Four instruments were scales. Surface instruments are only useful for grades I and II and not for grades III and IV PUs. Of the volume instruments, two filled the ulcer with saline or alginate. These are only useful for grades III and IV PUs, and the time needed to complete this test is prohibitive for a bedside test. The third, the three-dimensional

${ }^{1}$ Department of Spinal Cord Medicine, De Hoogstraat Rehabilitation, Utrecht, The Netherlands; ${ }^{2}$ Brain Centre Rudolf Magnus and Centre of Excellence for Rehabilitation Medicine, University Medical Centre Utrecht and De Hoogstraat Rehabilitation, Utrecht, The Netherlands and ${ }^{3}$ Department of Rehabilitation Medicine, Centre for Rehabilitation, University of Groningen, University Medical Centre Groningen, Groningen, The Netherlands

Correspondence: Dr FWA Van Asbeck, Department of Spinal Cord Medicine, De Hoogstraat Rehabilitation, Paranadreef 2, Utrecht NL3563 AZ, The Netherlands.

E-mail: fvasbeck@tiscali.nl

Received 8 December 2014; revised 29 January 2015; accepted 4 February 2015; published online 10 March 2015 
ruler instrument, is useful for grades I-IV surface or crater PUs, and the time needed seems reasonable. ${ }^{11}$ The disadvantage of the described instrument is that it cannot be used for undermining, tunneling or sinus tract formation. The four scales meeting the criteria were the Pressure Sore Status Tool (PSST), ${ }^{12}$ the Sessing scale, ${ }^{13}$ the Pressure Ulcer Scale for Healing (PUSH) ${ }^{14,15}$ and the DESIGN-R. ${ }^{10}$ Of these scales, only the PSST, in 2001 revised and renamed the Bates-Jensen Wound Assessment Tool (BWAT), ${ }^{16}$ assesses surface and undermining but with different instruments. During the current study, one instrument was described measuring the undermining with a cottontipped applicator swab, which is marked at the skin level and then compared with a ruler. After adding this to the depth, the current wound volume as percentage of the initial wound volume is calculated. ${ }^{17}$ This method was only found in one publication, and no clinimetric properties were given. The method needs calculation and is likely to give an overestimation of the wound volume.

A recent systematic review of instruments designed to measure the dimensions of PUs concluded that about three reliable methods for measuring PU surface area exist but that no conclusions could be reached about studies of diameter or depth. ${ }^{18}$

Thus, in the reviewed literature, we found no reliable method to predict the progress of healing in PUs using a wound measurement instrument that measures length, width, depth, undermining, tunneling or sinus tracts with one instrument. The aim of the current study is to describe the development of such an instrument that is practical at bedside, reliable, easy to learn, quick to perform and cheap, and to use this new instrument to predict the progress of healing of PUs in SCI patients.

\section{MATERIALS AND METHODS}

\section{Design}

This is a prospective cohort study. To make a prediction model for the progress of healing of PUs, 'Treatment Result Forms' of patients treated for PU were developed and completed on a weekly basis.

\section{Patients}

Included were adult SCI or spina bifida patients, treated as inpatients for grades II-IV PUs on sacrum or ischial tuberosity between 2001 and 2011. All patients were initially conservatively treated according to one guideline-based protocol for PU treatment in the 'cleaning phase', the 'granulation-epithelialization phase' and the 'crust phase'. Only patients with a granulation-epithelialization phase of 5 weeks or more were included.

Excluded were patients with PUs on other parts of the body or other primary neurological conditions.

\section{Instruments}

Building on the work of Kundin ${ }^{11}$ and Bates-Jensen, ${ }^{12}$ we developed the 'Decustick' to measure the length, width, depth, undermining, tunneling and sinus tract formation with one instrument, as recommended in the guidelines. ${ }^{1}$ This instrument is locally made, using a $15-\mathrm{cm}$ (6 inch) cotton swab on which centimeter marks were applied, with a double mark at 5 and $10 \mathrm{~cm}$. To make it reusable, a transparent disposable thermometer probe cover was used during measurement. According to the guideline, ${ }^{1}$ length and width measurements were taken from the intact skin edges. Depth and undermining, including sinus tract, measurements were taken from the deepest point to the imaginary surface in continuity with the edges of the wound. All measurements were taken weekly with the patient in the same position and rounded off upward to $0.5 \mathrm{~cm}$. A pilot study on the inter-rater reliability of the 'Decu-stick' based on 29 measurements in 7 patients showed excellent reliability with Spearman's correlations of 0.98 for the vertical dimension, 0.95 for the horizontal dimension and 0.99 for

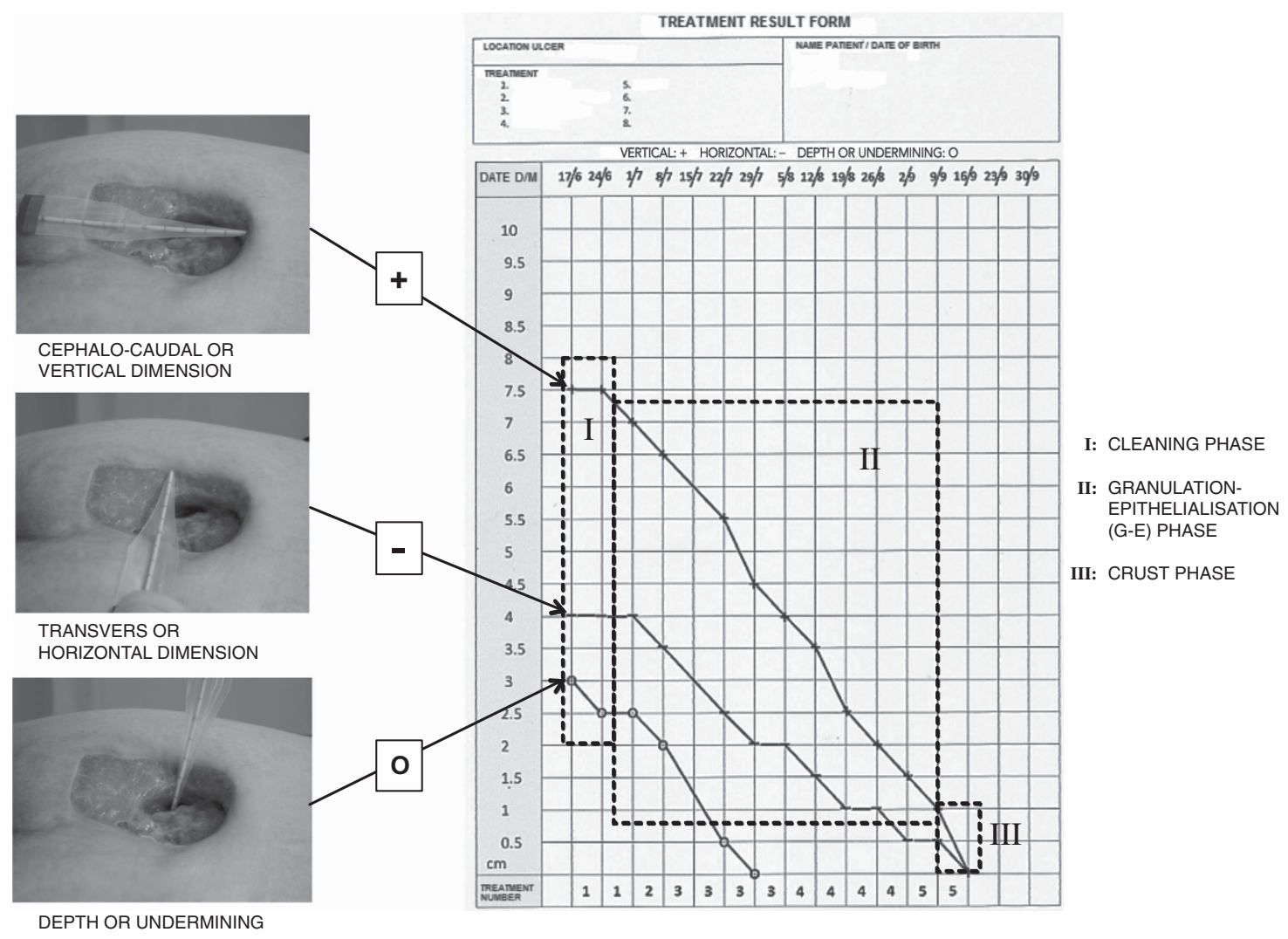

Figure 1 Measurement of length, width and depth of a PU, registered with a ' + ', a ' - ' and a 'o' on the 'Treatment Result Form'. The cleaning phase, granulation-epithelialization phase and crust phase are on this form. 
undermining. ${ }^{19}$ As PUs are typically irregularly shaped, computing the volume from this dimensions is flawed and instead these two- or three-dimensions are separately noted on a 'Treatment Result Form,' with the weeks on the $x$ axis starting with the date of the first measurement and the dimensions in $0.5 \mathrm{~cm}$ on the $y$ axis (Figure 1).

The greatest vertical (cephalo-caudal) surface dimension is noted with a ' + ', the horizontal (transversal) surface dimension, perpendicular to the vertical, is noted with a '-' and the depth, undermining or tunneling is noted with a ' $\mathrm{o}$ '. If the longest surface dimension is not exactly vertical or horizontal, the ' + ' is given to the dimension that is nearer to the vertical than to the horizontal and the ' -' is given to the dimension that is nearer to the horizontal than to the vertical. If in a week there happened to be no measurement, this week was left open and the next measurement was filled in the next week. The ' + 's were connected with the 't's, the '- 's with the '-'s and the 'o's with the 'o's in order to give a graphical impression of the progress of healing. The details on measurement and registration are also published elsewhere (www.decu-stick. org).

The lines in the graphs typically started 1-2 weeks horizontal, the cleaning phase (I), followed by gradually sloping down during the granulationepithelialization phase (II). In the last 1-2 weeks, there was usually a crust phase (III). Until the crust fell of and intact skin appeared, the wound could not be measured.

\section{Statistics}

We calculated the average decline of the greatest dimension of each ulcer in $\mathrm{cm} /$ week during the first 2, 3 and 4 weeks of the granulation-epithelialization phase to predict the average progress of healing from week 2, 3 and 4, respectively, until the end of observation. The end of observation was closure, operation or discharge with an open ulcer.

We calculated the prediction for this decline for the progress of healing with on average $<0.25,0.25-0.50,0.50-0.75$ and $>0.75 \mathrm{~cm}$ per week. From these calculations, the positive predictive value (PPV) and the negative predictive value (NPV) were established.

\section{RESULTS}

Forty-five patients with a total of 51 PUs were included. Forty-two patients had SCI (34 traumatic, 4 vascular, 2 transvers myelitis and 2 tumor) and 3 had spina bifida. Seventy-five percent of the patients were male, mean age was 55.5 (range 19-83) years, 71\% had a complete lesion ASIA Impairment Scale A (AIS A) and 22.2\% had a cervical lesion (C5-8). For the SCI patients, the mean time since injury was $11.2(0-54)$ years.

Of the PUs, 7 were grade II and 44 were grades III and IV. The greatest dimension was vertical in 27 ulcers $(1.5-10.5 \mathrm{~cm})$, horizontal in 11 ulcers $(3-15 \mathrm{~cm})$ and undermining in 13 ulcers $(4-12 \mathrm{~cm})$. Patients were followed up until closure after conservative treatment in 27 ulcers (5-31, mean 12.8 weeks), until operation in 18 ulcers (10-23, mean 14.8 weeks) or until discharge from the rehabilitation center with an open ulcer in 6 ulcers (12-37, mean 22.5 weeks).

To predict the average progress of healing $\geqslant$ or $<0.5 \mathrm{~cm}$ per week turned out the best of the calculated declines.

The PPV and NPV of the prediction of the progress of healing $\geqslant 0.5 \mathrm{~cm}$ per week were calculated with the data of the first 2,3 and 4 weeks. The prediction based on healing in the first 4 weeks was superior. The results are displayed in Table 1. The PPV was 95\% and the NPV was $96 \%$. The prediction based on the first 2 weeks of healing showed a PPV of $95 \%$ and an NPV of $62 \%$, and the prediction based on the first 3 weeks of healing showed a PPV of 95\% and an NPV of $78 \%$.

Figures 2 and 3 show the progress of healing during the first 4 weeks compared with the progress of healing from week 4 until the end of observations (see Figures 2 and 3 and Table 1).
Table 1 Number of ulcers with an average progress of healing $\geqslant$ or $<0.5 \mathrm{~cm}$ per week during weeks $1-4$ compared with the average progress of healing from week 4 until the end of observation

\begin{tabular}{|c|c|c|c|c|}
\hline & & \multicolumn{3}{|c|}{$\begin{array}{l}\text { Progress of healing during } \\
\text { weeks 1-4 }\end{array}$} \\
\hline & & $\begin{array}{l}\geqslant 0.5 \mathrm{~cm} \\
\text { per week }\end{array}$ & $\begin{array}{l}<0.5 \mathrm{~cm} \\
\text { per week }\end{array}$ & \\
\hline \multirow[t]{2}{*}{$\begin{array}{l}\text { Progress of healing from week } \\
4 \text { until the end of observation }\end{array}$} & $\geqslant 0.5 \mathrm{~cm}$ per week & 22 & 1 & 23 \\
\hline & $<0.5 \mathrm{~cm}$ per week & $\begin{array}{c}1 \\
23\end{array}$ & $\begin{array}{l}27 \\
28\end{array}$ & $\begin{array}{l}28 \\
51\end{array}$ \\
\hline
\end{tabular}

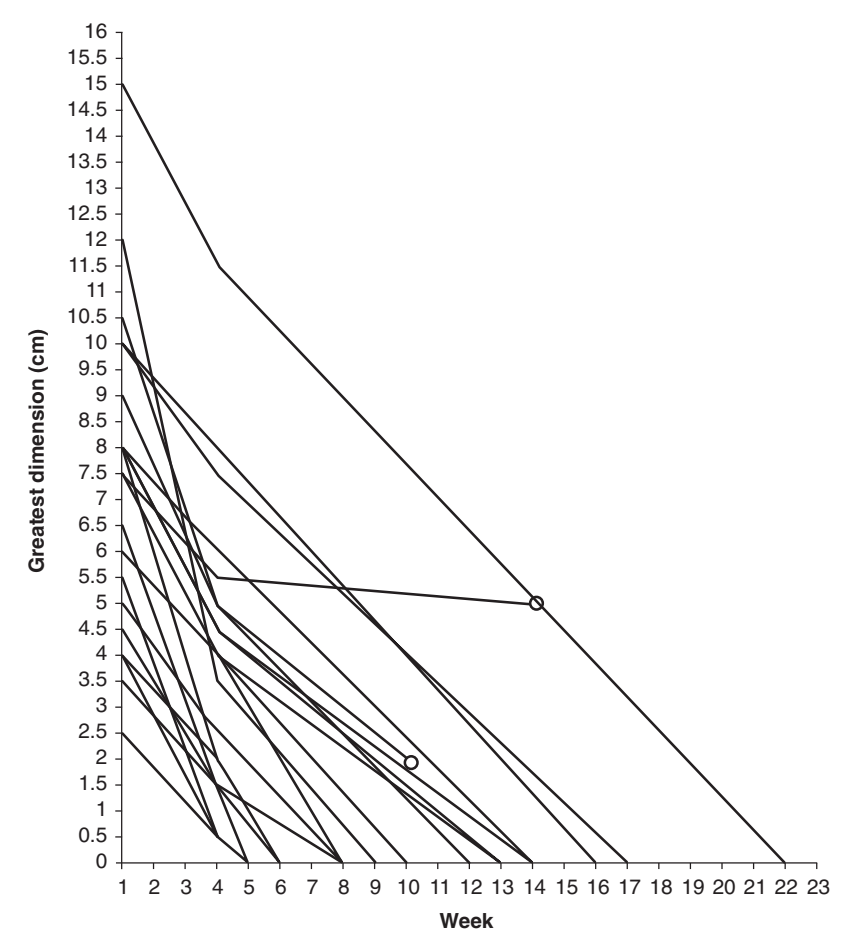

Figure 2 Average progress of healing of the greatest dimension in the first 4 weeks of the G-E phase of $\geqslant 0.5 \mathrm{~cm}$ per week in 23 patients, compared with the average progress of healing until the end of observation $(\mathrm{O}=$ operation $)$.

\section{DISCUSSION}

The results of this study show that the Decu-stick allows a simple and quick measurement of the length, width, depth and undermining of PU in patients with SCI without further calculation. This is an advantage, as undermining is present in the majority of PUs in our series, and this cannot be measured with plain or stereographic photography. In the literature, no instruments with comparable properties are described.

Further, this study demonstrated that after 4 weeks of conservative treatment a clinically useful prediction of the duration and success of conservative treatment can be made.

From the available measurements, we calculated the percentage of wound volume reduction as done by Barber, ${ }^{17}$ but we could not 


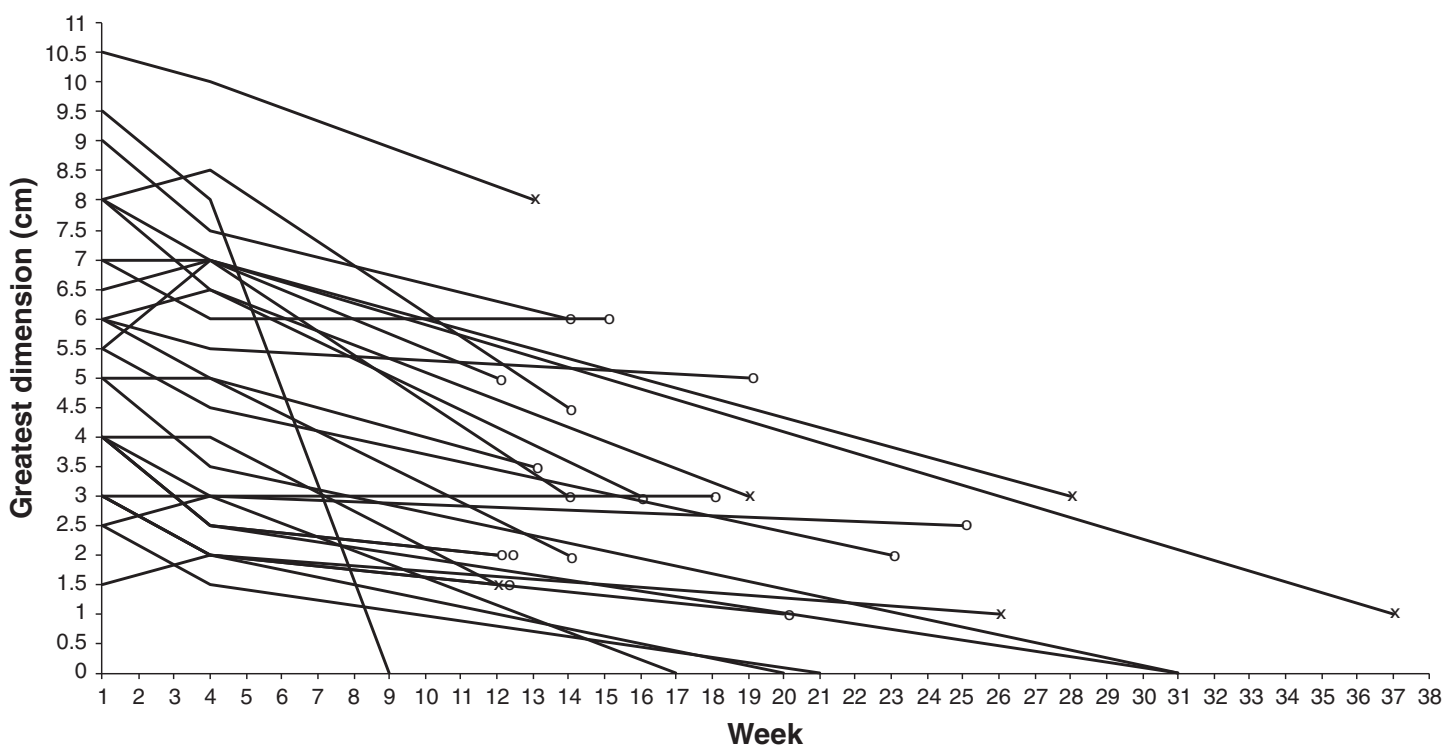

Figure 3 Average progress of healing of the greatest dimension in the first 4 weeks of the G-E phase of $<0.5 \mathrm{~cm}$ per week in 28 ulcers, compared with the average progress of healing until the end of observation $(\mathrm{O}=$ operation, $\mathrm{X}=$ discharged with open ulcer).

predict the progress of healing from these data (results not shown). The reason for this is not directly clear but we think it might be due to the overestimation of the volume calculation with this method. Therefore, we decided to work with the measured dimensions without further calculation of the wound volume.

The Decu-stick is a new bedside instrument to measure these dimensions with one instrument without the need to calculate wound volumes. It has the advantage that it is reliable, quick, cheap, easy to learn even for people without a medical or nursing background and the visual display provides appealing and useful feedback on the healing of the PU. Physicians and nurses can use this to monitor the healing process and for timely change of the treatment. This can also give clear feedback to the patient that can motivate him or her to adhere to the often annoying and lengthy conservative treatment. This instrument can also be used in the outpatient department or at home, where it can be used for treatment evaluation by e-health.

\section{Limitations of the study}

This study was conducted in one center with a limited number of patients with PUs. The conclusions heave to be supported by further studies with a bigger number of participants. It has to be established whether the progress of healing in PUs in other locations than sacrum and ischial tuberosity can be predicted with this method.

In surface ulcers, we considered whether the smallest dimension gives a better prediction than the greatest dimension. In our population, there were only five surface ulcers with a length and width with more than $1 \mathrm{~cm}$ difference. This number was too small to draw conclusions.

\section{CONCLUSION}

The 'Decu-stick' and the presented way to monitor the healing process provides a reliable, quick, cheap and easy-to-learn bedside method to measure the healing of PUs. It is the only method described so far to measure surface, crater, undermining, tunneling or sinus tract PUs with one instrument.
In conservative $\mathrm{PU}$ treatment, a progress of healing during the first 4 weeks of $\geqslant 0.5 \mathrm{~cm}$ per week of the greatest dimension as measured with the Decu-stick predicts the progress of healing with on average $\geqslant 0.5 \mathrm{~cm}$ per week from week 4 until the end of treatment, with a PPV of 0.95 and a NPV of 0.96 . This prediction can be used as preliminary evidence for the decision on operative or alternative conservative treatment.

\section{DATA ARCHIVING}

There were no data to deposit.

\section{CONFLICT OF INTEREST}

The first author is chairman of the non-profit foundation 'Meten is weten' (Measurement for knowledge) that has provided the instruction website www.decu-stick.org. The remaining authors declared no conflict of interest.

1 Consortium for Spinal Cord Medicine. Pressure Ulcer Prevention and Treatment Following Spinal Cord Injury: A Clinical Practice Guideline for Health-Care Professionals. Paralysed Veterans of America: Washington, DC, USA, 2000.

2 Raghavan P, Raza WA, Ahmed YS, Chamberlain MA. Prevalence of pressure sores in a community sample of spinal injury patients. Clin Rehabil 2003; 17: 879-884.

3 Nogueira PC, Caliri MH, Haas VJ. Profile of patients with spinal cord injuries and occurrence of pressure ulcer at a university hospital. Rev Lat Am Enfermagem 2006, 14: 372-377.

4 Verschueren JH, Post MW, de Groot S, van der Woude LH, van Asbeck FW, Rol M et al. Occurrence and predictors of pressure ulcers during primary in-patient spinal cord injury rehabilitation. Spinal Cord 2011; 49: 106-112.

5 Xu Y, Sun J, Carter RR, Bogie KM. Personalized prediction of chronic wound healing: an exponential mixed effects model using stereophotogrammetric measurement. J Tissue Viability 2014; 23: 48-59.

6 Flanagan M. Improving accuracy of wound measurement in clinical practice. Ostomy Wound Manage 2003; 49: 28-40.

7 Jessup RL. What is the best method for assessing the rate of wound healing? A comparison of 3 mathematical formulas. Adv Skin Wound Care 2006; 19 138-147.

8 Landelijke Richtlijn Preventie en Behandeling Decubitus. Verpleegkundigen \& Verzor genden Nederland (V\&VN) (National Guideline Prevention and Treatment Pressure Ulcers. Dutch Nursing Association): Utrecht, The Netherlands, 2011, pp 19.

9 Van Lis MS, van Asbeck FW, Post MW. Monitoring healing of pressure ulcers: a review of assessment instruments for use in the spinal cord unit. Spinal Cord 2010; 48: 92-99. 
10 Matsui Y, Furue M, Sanada H, Tachibana T, Nakayama T, Sugama J et al. Development of the DESIGN-R with an observational study: an absolute evaluation tool for monitoring pressure ulcer wound healing. Wound Repair Regen 2011; 19: 309-315.

11 Kundin JI. A new way to size up a wound. Am J Nurs 1989; 89: 206-207.

12 Bates-Jensen BM. The Pressure Sore Status Tool, a few thousand assessments later. Adv Wound Care 1997; 10: 65-73.

13 Ferrell BA. The Sessing Scale for measurement of pressure ulcer healing. Adv Wound Care 1997; 10: 78-80.

14 Sussman C. Presenting a draft pressure ulcer scale to monitor healing. Adv Wound Care 1997; 10: 92.

15 Stotts NA, Rodeheaver GT, Thomas DR, Frantz RA, Bartolucci AA, Sussman C et al. An instrument to measure healing in pressure ulcers: development and validation of the
Pressure Ulcer Scale for Healing (PUSH). J Gerontol A Biol Sci Med Sci 2001; 56: M795-M799.

16 Harris C, Bates-Jensen B, Parslow N, Raizman R, Singh M, Ketchen R et al. BatesJensen wound assessment tool: pictorial guide validation project. J Wound Ostomy Continence Nurs 2010; 37: 253-259.

17 Barber S. A clinically relevant wound assessment method to monitor healing progression. Ostomy Wound Manage 2008; 54: 42-49.

18 O'Meara SM, Bland JM, Dumville JC, Cullum NA. A systematic review of the performance of instruments designed to measure the dimensions of pressure ulcers. Wound Repair Regen 2012; 20: 263-276.

19 Asbeck FWA van. De DECU-stick: een eenvoudige manier om decubitus te meten. Ned Tijdschr Geneesk 1997; 141: 1718. 